\title{
A Hybrid Vibration Powered Microelectromechanical Strain Gauge
}

\author{
Yu Jia, Cuong D. Do, Xudong Zou and Ashwin A. Seshia \\ Nanoscience Centre, University of Cambridge, Cambridge CB3 0FF, UK \\ E-mail: yu.jia.gb@ieee.org, cdd38@cam.ac.uk, xz280@cam.ac.uk, aas41@cam.ac.uk
}

September 14, 2015

\begin{abstract}
This paper reports the demonstration of an ultra-low power MEMS-CMOS oscillator for strain sensing, powered by a miniature piezoelectric vibration energy harvester (VEH). The employment of the Pierce oscillator topology in a MEMS-CMOS oscillator allows for minimisation of the power requirement to as low as 1.1 $\mu \mathrm{W}$ under ideal conditions. A VEH prototype, developed with hard PZT on a stainless steel substrate $\left(\sim 0.4 \mathrm{~cm}^{3}\right.$ practical operational volume), is able to deliver a typical average power of $187 \mu \mathrm{W}$ at $11.4 \mathrm{~ms}^{-2}$ and $514 \mathrm{~Hz}$. Some of the practical challenges associated with the integration of the harvester and the MEMS sensor have also been explored, which helps to lay the foundation for realising net-zero-power strain sensors.
\end{abstract}

Keywords: piezoelectric, vibration energy harvesting, MEMS Strain Gauge, CMOS Oscillator

\section{Introduction}

Autonomous self-powered sensor systems are of increasing interest for a variety of contexts in wireless condition and structural health monitoring applications [1]. Some of the key enabling components for these self-sustaining systems include the development of low power oscillator circuits for sensors and real-time clock applications, low power MEMS (micro-electromechanical system) sensors for measurement of environmental conditions, high power density energy harvesters and efficient power conditioning and power management systems. There is a potential to realise a self-sustaining smart monitoring system when these elements are combined. This paper demonstrates an ultra-low power MEMS resonant strain sensor enabled by a miniature piezoelectric vibration energy harvester; and explores some of the integration challenges involved.

MEMS resonators have been previously employed as transducers in accelerometers, gyroscopes, strain sensors, electrometers, mass sensors etc. The advantage of these MEMS sensors include low power consumption, high sensitivity and potential economies of scale similar to conventional semiconductors. Additionally, the possibility of low power front-end interface circuit $[2,3]$ for these resonators has broad applicability to a variety of sensing contexts. This would enable ultra low power operation for both the MEMS sensors as well as the interface circuit in order to read out useful sensor measurements.

Amongst the three major mechanical-toelectrical transduction mechanisms, piezoelectric transducers are most suitable for miniature vibration energy harvesting $(\mathrm{VEH})$ in order to recover ambient kinetic energy [4]. This is due to the low power density of its electrostatic counterparts as well as the poor downwards scaling of electromagnetic generators. The current state-of-the-art piezoelectric $\mathrm{VEH}$ has been reported to provide peak power of up to a few hundreds of microwatts per centimetre cube, per g of acceleration; while PZT (lead zirconate titanate) is the most popular bulk piezoelectric material due to its high charge constant $[4,5]$.

For MEMS piezoelectric VEH, AlN and $\mathrm{ZnO}$ have been the conventional choice despite their lower strain constants $[5,6]$, due to complexity in fabrication for incorporating PZT in MEMScompatible fabrication process $[7,8]$. Nonetheless, sol-gel PZT [7], deposited aerosol PZT [9] or thinned bulk PZT [10] have witnessed integration into either MEMS fabrication or miniature assembly. This paper reports the investigation of incorporating bulk soft piezoelectric ceramic and hard piezoelectric ceramic onto a stainless steel substrate in order to realise a miniature high power density vibration energy harvester suitable for sustaining MEMS sensors.

From the literature, integrated vibration powered wireless sensor systems have been demonstrated with both miniature electromagnetic VEH [11] and MEMS piezoelectric VEH [12]. Additionally, certain sensing systems such as temperature sensors and acceleration sensors have been incorporated into such VEH-enabled wireless systems [13]. 
The development of self powered strain sensors in particular, for structural health monitoring applications, have been highlighted for ensuring the operational safety and asset management of the civil infrastructure $[14,15]$. However, conventional foil gauges and vibrating wire gauges typically consume electrical power in the order of 100's mW, which significantly diverges from what is practically achievable by the state-of-the-art of VEH [5].

While previous literature on self-powered strain sensors can be found $[15,16]$, these systems have been designed as a basic piezoelectric resonator acting as both the generator and the sensor, which lacks the sensitivity and reliability of dedicated strain sensors. By combining both the ultra low power operation of MEMS-CMOS oscillators for strain sensing and miniature piezoelectric vibration energy harvesting, an integrated self-powered high accuracy strain sensor can thus be realised. The device reported in this paper demonstrates a sensor that consumes several orders of magnitude lower power than the most power efficient foil gauges [17], while achieving several orders of magnitude higher sensitivity than the most sensitive foil gauges [18]; thus, making it possible to sustain the operation of the strain measurement system purely by ambient kinetic energy recovered by a miniature vibration energy harvester.

\section{Vibration Energy Harvester}

The harvester $\left(\sim 0.4 \mathrm{~cm}^{3}\right.$ practical volume), with the stack of material outlined in figure 1 , was constructed from a PZT square plate of $10 \mathrm{~mm}$ side length and $0.25 \mathrm{~mm}$ thickness. Practical volume is defined by the volume required to accommodate the oscillatory vibration of the harvester. The final prototype consisted of a layer of brittle PZT attached to a $50 \mu \mathrm{m}$ thick stainless steel substrate in order to enhance its ability to accumulate high strain energy without fracturing. An estimated 1.7 grams of solder was added as the proof mass to improve the power responsiveness.

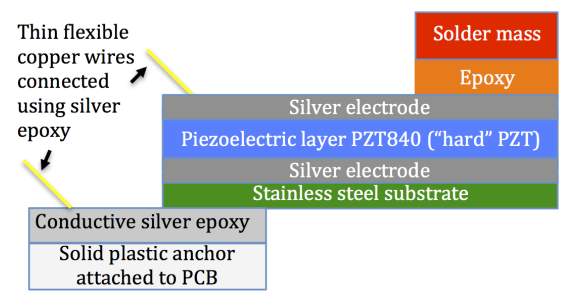

Figure 1: Stack of material for PZT vibration energy harvester.

The natural frequency $\omega_{0}$ for a cantilever beam with added mass can be defined by equation 1 [19].

$$
\omega_{0}^{2}=\frac{E w h^{3}}{4\left(0.24 m_{b}+m_{l}\right) l^{3}}
$$

where, $k$ is the stiffness constant, $m$ is the effective seismic mass of the resonator, $E$ is the elastic modulus, $w$ is the width of the cantilever, $h$ is the effective thickness of the composite beam, $m_{b}$ is the mass of the beam, $m_{l}$ is the end mass and $l$ is the effective length of the cantilever resonator.

Piezoelectric layers, when mechanically strained, induces electrical charges $q$, which is represented in equation 2 and the attainable power can be estimated by equation 3 [20].

$$
\begin{gathered}
q=d_{31} \varepsilon_{a v} E a_{p z} \\
P=\frac{\omega h_{p} q^{2}}{\varepsilon_{0} \varepsilon_{r} a_{p z}}
\end{gathered}
$$

where, $d_{31}$ is the piezoelectric charge constant in the 31 mode, $\varepsilon_{a v}$ is the average strain experienced by the piezoelectric transducer, $E$ is the elastic modulus of the piezoelectric material, $a_{p z}$ is the active piezoelectric area, $P$ is the peak power, $\omega$ is the frequency, $h_{p}$ is the thickness of the piezoelectric layer, $\varepsilon_{0}$ is the permittivity of free space and $\varepsilon_{r}$ is the dielectric constant of the piezoelectric material.

Two types of PZT materials from APC International Ltd. were investigated for suitability as a vibration energy harvester. One is a soft piezoelectric ceramic PZT-855, also dubbed as Navy VI and the other is hard piezoelectric ceramic PZT-840, also dubbed as Navy I. Selected properties of the two piezoelectric materials are listed in table 1 .

It can be seen that PZT-855 has a significantly higher charge constant than PZT-840. Therefore charge generated per newton of force for PZT-855 should theoretically be higher. However, PZT-840 is more brittle than PZT-855 and therefore fractures at smaller strain levels. However, in the absence of a substrate material, both the soft and hard PZT are too brittle for the purpose of vibration energy harvesting. PZT-855 easily fractures during handling in the absence of a substrate. PZT-840 without an additional substrate layer fractured when subjected to less than $0.1 \mathrm{~g}$ of vibration. Even with the addition of a stainless steel substrate, PZT-855 cracked on the substrate. Figure 2 shows these 3 preliminary iterations of cantilever prototypes that were not sufficiently robust for the purpose of vibration energy harvesting ( $\mathrm{VEH})$.

Figure 3 presents the final iteration of the miniature piezoelectric VEH prototype that incorporated the $250 \mu \mathrm{m}$ hard PZT-840 onto a $50 \mu \mathrm{m}$ stainless steel substrate. Approximately $2 \mathrm{~mm}$ of the square PZT plate was sacrificially overlapped onto the substrate as part of the anchor. This resultant device remained intact within the tested acceleration 
Table 1: Selected properties of the two PZT materials chosen in this study.

\begin{tabular}{l|ccc} 
Property & PZT-840 (hard) & PZT-855 (soft) & unit \\
\hline Relative dielectric constant $\varepsilon_{r}$ & 1275 & 3300 & \\
Electromechanical coupling factor $k_{31}$ & 0.35 & 0.40 & \\
Piezoelectric charge constant $-d_{31}$ & 125 & 276 & $\mathrm{pC} / \mathrm{N}$ \\
Piezoelectric voltage constant $-g_{31}$ & 11.0 & 9.0 & $\mathrm{mVm} / \mathrm{N}$ \\
Elastic modulus $E$ & 80 & 59 & $\mathrm{GPa}$
\end{tabular}

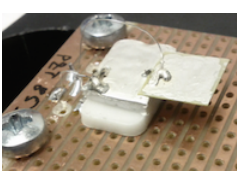

(a) PZT-855 (soft piezoelectric) without substrate

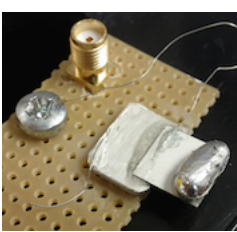

(c) PZT-855 on stainless steel substrate

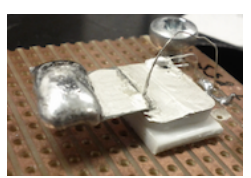

(hard piezoelec-

tric) without

substrate

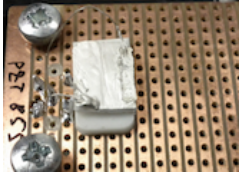

(d) An example of fractured device (b) PZT-840

Figure 2: Preliminary iterations of piezoelectric vibration energy harvester prototypes. The use of soft piezoelectric ceramic (either with or without substrate) and the use of hard piezoelectric ceramic without substrate, resulted in brittle fracture either during handling or when subjected to very small vibrational input $(<0.1$ g).

range of up to $\sim 1.2 \mathrm{~g}$ of vibration at its fundamental mode resonant frequency.

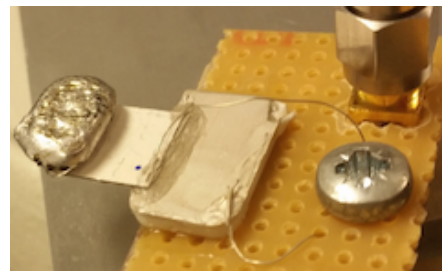

Figure 3: Final piezoelectric vibration energy harvester prototype using PZT-840 on stainless steel substrate.

An off-the-shelf power conditioning board was employed to rectify and regulate the harvester AC power: EHE004 from MIDE Technology, which incorporates LTC3588-1 from Linear Technology and has a $200 \mu \mathrm{F}$ on-board storage capacitor. The power conditioning board was configured to deliver a regulated $1.8 \mathrm{~V}$ DC supply $\left(V_{d c}\right)$ when the voltage across the storage capacitor $\left(V_{c a}\right)$ has charged up to $4.0 \mathrm{~V}$. This regulated supply can be maintained until $V_{\text {cap }}$ drops below $3.0 \mathrm{~V}$.

Experimental power output values of the $\mathrm{VEH}$ are given in figure 4 and table 2 . The peak and average power were measured across a matched resistance of $80 \mathrm{k} \Omega$ at the natural frequency of 514 $\mathrm{Hz}$. By varying the resistive load feeding off the regulated $1.8 \mathrm{~V}$ supply from the EHE004 and monitoring the voltage variations across the storage capacitor, the conditioned power was determined.

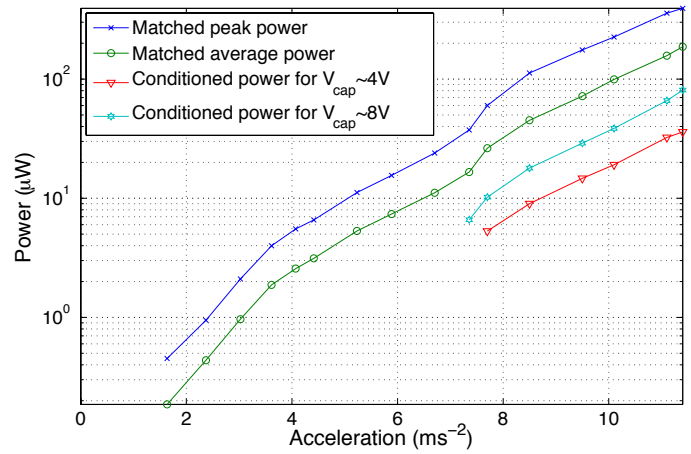

Figure 4: Power output versus acceleration for the PZT harvester.

On average, when the voltage across the storage capacitor was in the range of $3.5 \mathrm{~V}$ to $4.5 \mathrm{~V}$, the power conversion efficiency (conditioned power/matched average power) was in the order of $20 \%$. This efficiency increased up to $40 \%$ when the $V_{c a p}$ was in the vicinity of $8.0 \mathrm{~V}$. This demonstrated the effect of varying impedance as the storage capacitor is charged up. However, a significant proportion of the power is lost due to both unmatched impedance in the power conditioning circuit and storage as well as the diode threshold required to operate the full bridge rectifier in the LTC3588-1 chip. The later can be evidenced by the zero output conditioned power at low acceleration levels when the harvester voltage $V_{a c}$ does not attain the bare minimum rectifier diode threshold.

\section{MEMS Strain Gauge}

A micro-fabricated double-ended tuning fork (DETF) resonator, based on previous work from the group $[21,22,23,24]$ and illustrated in figure 5, was employed as the strain gauge. The MEMS chip, 
Table 2: Experimentally measured harvester power across a matched load and from a power conditioning circuit at various storage capacitor voltage.

\begin{tabular}{l|c|cc|cc}
$\begin{array}{l}\text { Acc. } \\
\left(\mathrm{ms}^{-2}\right)\end{array}$ & $\begin{array}{c}\text { Matched average } \\
\text { power }(\mu \mathrm{W})\end{array}$ & $\begin{array}{c}\text { Conditioned power } \\
\text { for } V_{\text {cap }} 4 V(\mu \mathrm{W})\end{array}$ & $\begin{array}{c}\text { Efficiency } \\
(\%)\end{array}$ & $\begin{array}{c}\text { Conditioned power } \\
\text { for } V_{\text {cap }} 8 V(\mu \mathrm{W})\end{array}$ & $\begin{array}{c}\text { Efficiency } \\
(\%)\end{array}$ \\
\hline 6.7 & 11.1 & 0 & 0 & 0 & 0 \\
7.4 & 16.6 & 0 & 0 & 6.6 & 40 \\
7.7 & 26.3 & 5.3 & 20 & 10.2 & 39 \\
8.5 & 45.1 & 9.0 & 20 & 17.9 & 40 \\
9.5 & 72.0 & 14.7 & 20 & 29.0 & 40 \\
10.1 & 99.4 & 19.1 & 19 & 38.6 & 39 \\
11.1 & 157.5 & 32.4 & 21 & 66.0 & 42 \\
11.4 & 187.2 & 36.0 & 19 & 81.0 & 43
\end{tabular}

packaged within a chip carrier, was attached to the test beam of a strain calibration apparatus using an adhesive. The test apparatus was designed based on [22] and is illustrated in figure 6 . As the strain of the test beam is varied by a micrometere, the transferred strain to the DETF structure induces a shift in its resonant frequency. Therefore, through measuring the frequency shift of the DETF resonator and calibrating the strain sensitivity of the MEMS sensor, the applied mechanical strain can be extrapolated.

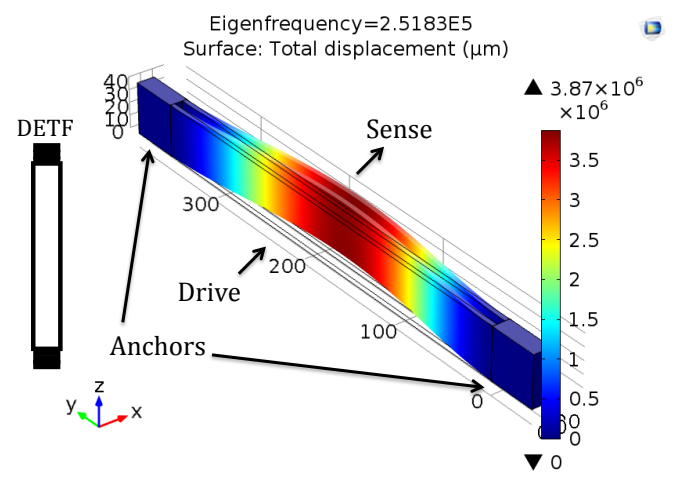

Figure 5: Design model of the double ended tuning fork resonating around $250 \mathrm{kHz}$.

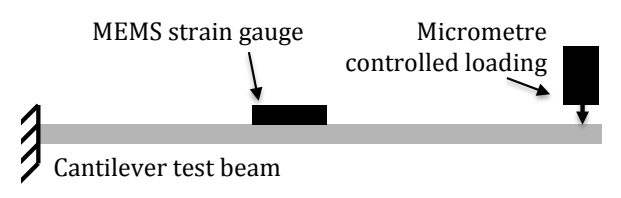

Figure 6: Schematic of the strain calibration apparatus.

The DETF resonators reported in this paper consists of two identical beams with dimensions of 350 $\mu \mathrm{m}$ in length, $\sim 4 \mu \mathrm{m}$ in width and $40 \mu \mathrm{m}$ in thickness. Parallel-plate electrostatic transducers with $2 \mu \mathrm{m}$ electrode gap are utilised for driving and sensing. The resonators are fabricated on a SOI wafer and are integrated into a wafer-level vacuum package. The DETF resonator is driven using one parallel-plate electrode and the motional current is measured from the other electrode by a transimpedance amplifier. The measured quality factor is approximately 16,000 .

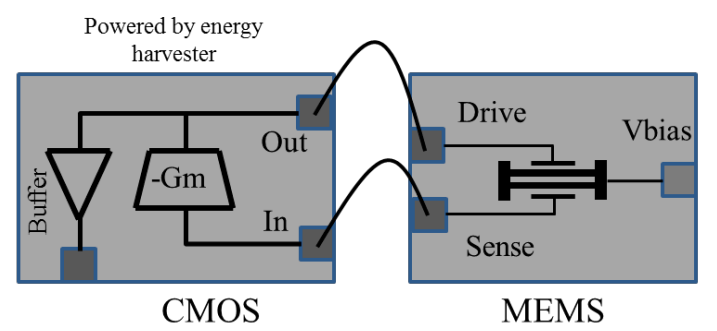

Figure 7: Schematic of the MEMS-CMOS oscillator for strain sensing.

The MEMS-CMOS oscillator is based on a Pierce topology due to its simplicity and potential for power minimisation with gain provided by a single transistor [3]. Oscillator interface circuit was fabricated in a $0.35 \mu \mathrm{m}$ CMOS technology and electrically packaged together with the DETF resonator as shown in figure 7 . The applied mechanical strain onto the structure to which the MEMS sensor is attached, translates to a frequency signal output from interface circuit. Therefore, the resultant shift in resonant frequency can be monitored.

Figure 8 illustrates a typical frequency-strain relationship characterised using the strain calibration apparatus. The deflection induced by the micrometre was used to analytically calculate the bending strain at the particular point along the cantilever beam.

The power requirement of the MEMS-CMOS oscillator is shown in table 3 . Although the devices themselves consumes only $1.89 \mu \mathrm{W}$ at a supply voltage of $1.8 \mathrm{~V}$, a significant amount of power is practically dissipated by the load capacitance of the cabling required to monitor and read out the frequency signal on the oscilloscope. The exact additional power dissipation from this parasitic source depends on the length (capacitance) of the cable. However, for a future iterations of integrated sys- 


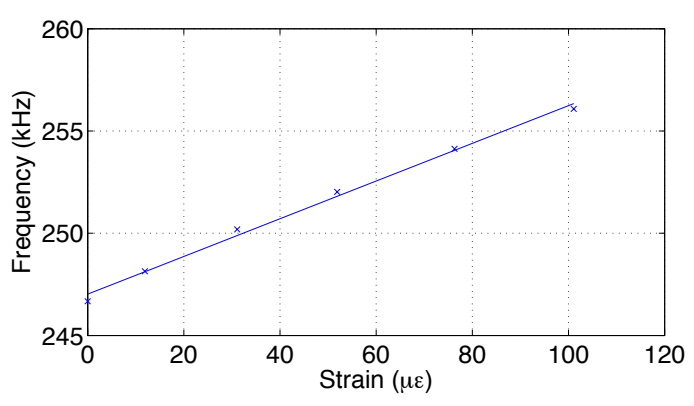

Figure 8: Frequency-strain characterisation of the MEMS strain gauge: $\sim 92 \mathrm{~Hz} / \mu \varepsilon$.

tem with on-board sensor readout, this issue can be minimised from the reduced loading.

Table 3: Power requirement of MEMS-CMOS oscillator with varying load capacitors at the output.

\begin{tabular}{r|cc} 
Load capacitance & \multicolumn{3}{|c}{ Power required $(\mu \mathrm{W})$} \\
$(\mathrm{pF})$ & $V_{d c}$ at $1.2 \mathrm{~V}$ & $V_{d c}$ at $1.8 \mathrm{~V}$ \\
\hline 0 & 1.08 & 1.89 \\
1 & 1.25 & 3.71 \\
5 & 1.88 & 4.91 \\
10 & 2.66 & 6.57 \\
50 & 9.0 & 20.5 \\
100 & 16.9 & 38.2 \\
150 & 24.8 & 55.9
\end{tabular}

\section{Integration}

The MEMS-CMOS oscillator, PZT VEH and the power conditioning board are shown in figure 9 . The experimental apparatus used for the integration test is outlined in figure 10. The harvester was driven by a vibration shaker while the MEMS strain gauge was attached to a beam whose mechanical strain can be altered by the strain calibration apparatus shown in figure 6 .

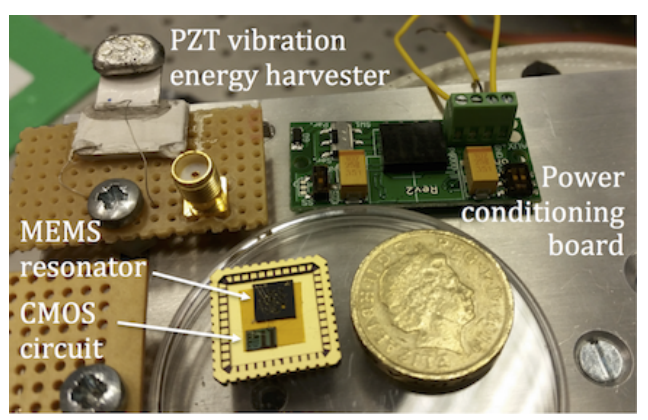

Figure 9: Photograph of MEMS-CMOS oscillator and PZT vibration energy harvester prototype.

Despite the notably diminished power efficiency from the off-the-shelf power conditioning circuit as shown in table 2, the conditioned power levels

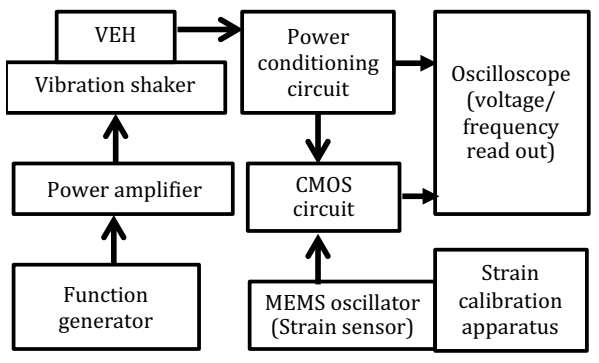

Figure 10: Schematic of the experimental apparatus used to characterise the vibration-powered MEMS strain sensor.

achievable at around $1 \mathrm{~g}$ of acceleration is already sufficient to practically sustain the MEMS-CMOS oscillator, while also accounting for the significant additional power drain due to the cable loading required to monitor the sensor output on an oscilloscope (table 3). Figure 11 illustrates an example where the MEMS-CMOS oscillator switches on when the storage capacitor in the power conditioning circuit has been charged up by the VEH; and when the sensor system switches off as the vibrational input is removed and the storage capacitor is drained.

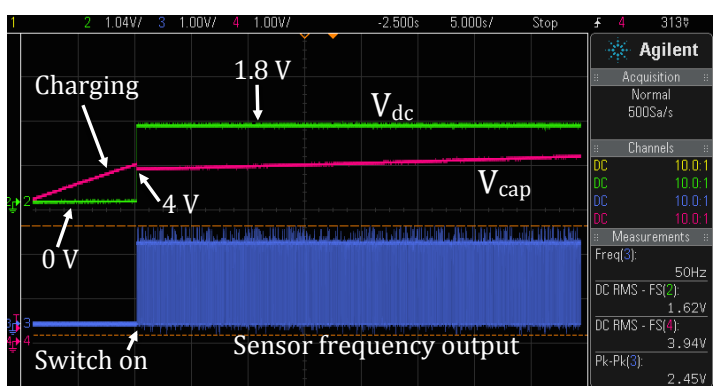

(a) Powering up

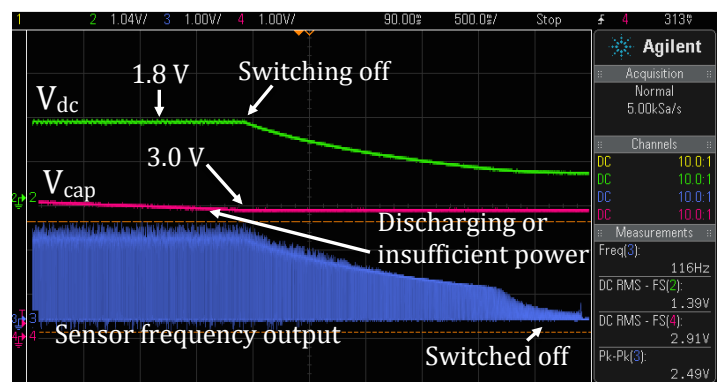

(b) Powering down

Figure 11: Charging up (switching on) and discharging (switching off) of the power conditioning circuit by vibration energy harvesting and MEMS-CMOS oscillator for strain sensing.

A dip in $V_{\text {cap }}$ can be seen as the MEMS-CMOS oscillator is switched on (figure 11a) and starts to drain power from the $1.8 \mathrm{~V}$ DC source provided by the power conditioning circuit. In this instance, the conditioned power harvested from the vibration 
input is more than the power usage of the MEMSCMOS oscillator (including the additional power dissipation from cable loading). Therefore, the $V_{\text {cap }}$ continues to increase, albeit at a slower rate than before the sensor was switched on.

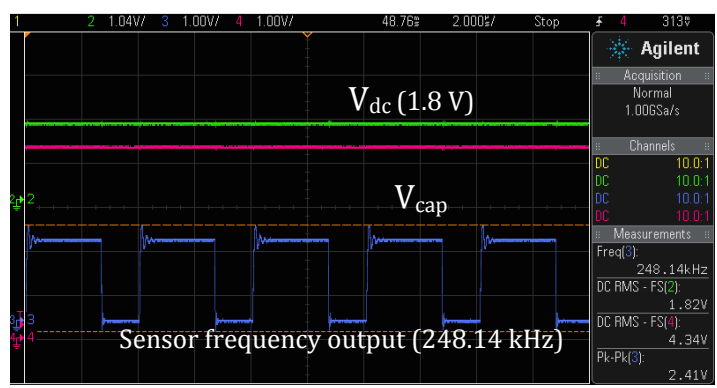

(a) $11.9 \mu \varepsilon$

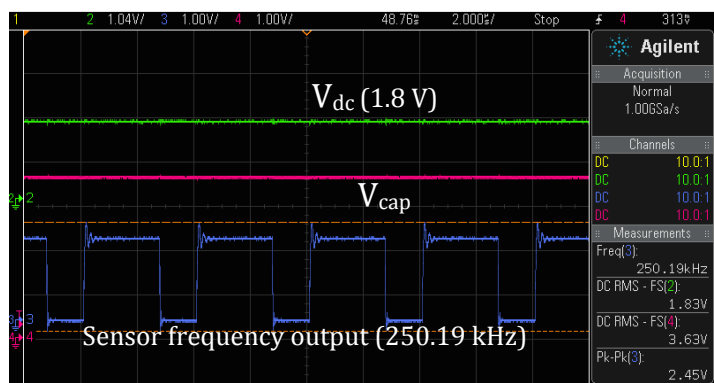

(b) $\sim 31.1 \mu \varepsilon$

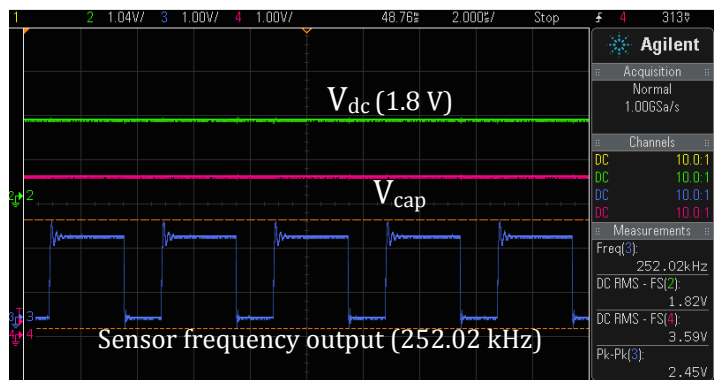

(c) $\sim 51.9 \mu \varepsilon$

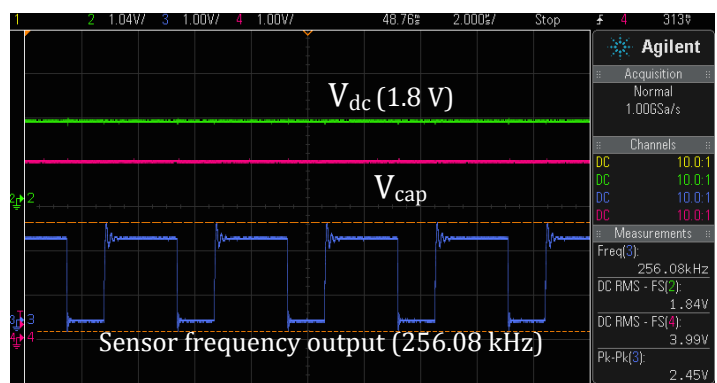

(d) $\sim 101.1 \mu \varepsilon$

Figure 12: Vibration energy harvester powered MEMS strain gauge showing various sensor readings as the beam to which the gauge is attached to is strained. Strain sensitivity is $\sim 90 \mathrm{~Hz} / \mu \varepsilon$

As the $V_{\text {cap }}$ falls below $3 \mathrm{~V}, V_{d c}$ is no longer able to sustain the $1.8 \mathrm{~V}$ regulated DC supply and voltage levels off towards zero. At first, the MEMS-CMOS oscillator remains functional (fre- quency output), while its own signal voltage also decreases corresponding to its supply voltage $V_{d c}$. However, the sensor signal is switched off after $V_{d c}$ drops below $\sim 0.6 \mathrm{~V}$.

Figure 12 expands on figure 11 during operating conditions and illustrates examples of the vibration powered MEMS strain sensor with varying strain conditions on the beam structure within the strain calibration apparatus. The MEMS oscillator was calibrated to have a sensitivity of $\sim 92 \mathrm{~Hz}$ per microstrain. The variation in the induced strain in the test beam, manually operated through the strain calibration apparatus, was successfully monitored by the vibration powered MEMS strain sensor.

Channel 2 shows the regulated $1.8 \mathrm{~V}$ DC output supplied from the power conditioning circuit to the MEMS-CMOS oscillator. Channel 4 monitors the voltage across the storage capacitor $V_{c a p}$. Channel 3 outputs the signal from the MEMS-CMOS oscillator. The frequency of the Channel 3 signal corresponds to the resonant frequency of the double-ended tuning fork resonator under a particular strain condition. Taking $246.67 \mathrm{kHz}$ as the starting zero strain condition, various strain gauge measurements were taken as shown in the figures.

\section{Conclusion and future work}

This paper has demonstrated a MEMS strain gauge and CMOS interface circuit powered up by a miniature piezoelectric vibration energy harvester (VEH). Despite the reduced power efficiency of the off-the-shelf power conditioning circuit $(81 \mu \mathrm{W}$ from $187 \mu \mathrm{W}$ at $11.4 \mathrm{~ms}^{-2}$ ), the conditioned power from the VEH was sufficient to power up the ultralow power sensor $(1.89 \mu \mathrm{W})$ as well as account for the significant additional power dissipation from cable loading (as high as $\sim 60 \mu \mathrm{W}$ ).

Future work will involve the optimisation of VEH parameters to increase the attainable power level; as well as potential incorporation of various nonlinear vibration phenomena $[25,26]$, to maximise the power efficiency from broadband vibration that is more representative of a larger variety of environments requiring structural health monitoring. The development of dedicated power conditioning circuits are required to improve the conversion efficiency of the harvested raw AC power into a stable DC source. Furthermore the supply voltage and loading capacitance can be minimised by boardlevel and chip-level integration, in order to reduce the actual power consumption by the circuit. 


\section{Acknowledgement}

This work was supported by EPSRC (grants: $\mathrm{EP} / \mathrm{K} 000314 / 1$ and EP/L010917/1).

\section{References}

[1] T. Kazmierski and S. Beeby, Eds., Energy Harvesting Systems Principles, Modeling and Applications. Springer US, 2011.

[2] K. Cioffi and W. Su, "32 khz mems-based oscillator for low power application," Freq. Control Sym. Expo., pp. 551-558, 2005.

[3] C. Do, A. Erbes, J. Yan, and A. Seshia, "Low power mems oscillators for sensor applications," in in Digest Tech. Papers EFTF, 2014, pp. 427-430.

[4] S. Beeby, M. Tudor, and N. White, "Energy harvesting vibration sources for microsystems applications," Meas. Sci. Technol., vol. 17, no. 12, pp. R175-R195, 2006.

[5] S. Priya and D. Inman, Energy Harvsting Technologies. New York: Springer US, 2009.

[6] A. Erturk and D. Inman, Piezoelectric energy harvesting. New Delhi, India: Wiley, 2011.

[7] N. Besse, A. Quintero, D. Briand, P. Janphuang, R. Lockhart, J. Ruan, and N. de Rooij, "Pzt-based energy harvesters on plastic foil optimized through theoretical modeling and fabrication improvements," in PowerMEMS, Atlanta, Georgia, USA, Dec. 02 - 05 2012, pp. pp. 42-45.

[8] R. Andosca, T. G. McDonald, V. Genova, S. Rosenberg, J. Keating, C. Benedixen, and $\mathrm{J}$. Wu, "Experimental and theoretical studies on mems piezoelectric vibrational energy harvesters with mass loading," Sens. Actuators A, vol. 178, pp. 76-87, 2012. [Online]. Available: http://dx.doi.org/10. 1016/j.sna.2012.02.028

[9] S. Lin and W. Wu, "Piezoelectric micro energy harvesters based on stainless-steel substrates," Smart Mater. Struct., vol. 22, p. 11, 2013.

[10] E. Aktakka, R. Peterson, and K. Najafi, "Thinned-pzt on soi process and design optimization for piezoelectric inertial energy harvesting," in Transducers, Beijing, China, June 5-9 2011, pp. pp. 1649-1652.

[11] R. Torah, P. Glynne-Jones, M. Tudor, T. O'Donnell, S. Roy, and S. Beeby, "Selfpowered autonomous wireless sensor node using vibration energy harvesting," Meas. Sci. Technol., vol. 19, p. 8pp, 2008.
[12] R. Elfrink, V. Pop, D. Hohlfeld, T. M. Kamel, S. Matova, C. de Nooijer, M. Jambunathan, M. Goedbloed, L. Caballero, M. Renaud, J. Penders, and R. van Schaijk, "First autonomous wireless sensor node powered by a vacuum-packaged piezoelectric mems energy harvester," in IEDMO9, Baltimore, MD, 7-9 Dec. 2009, pp. 22.5.1-4.

[13] S. George, "Development of a vibrationpowered wireless temperature sensor and accelerometer for health monitoring," in Aerospace Conference, 2006 IEEE, Big Sky, MT, 2006.

[14] E. Sazonov, H. Li, D. Curry, and P. Pillay, "Self-powered sensors for monitoring of highway bridges," IEEE Sensors Journal, vol. 9, no. 11, pp. 1422-1429, 2009.

[15] N. G. Elvin, N. Lajnef, and A. A. Elvin, "Feasibility of structural monitoring with vibration powered sensors," Smart Mater. Struct., vol. 15, pp. 977-986, 2006.

[16] N. G. Elvin, A. A. Elvin, and M. Spector, "A self-powered mechanical strain energy sensor," Smart Mater. Struct., vol. 10, pp. 293-299, 2001.

[17] T. Nagatama, M. Ruz-Sandoval, B. F. Spencer, K. A. Mechitov, and G. Agha, "Wireless strain sensor development for civil infrastructure," Trans. Society Instrument Control Engineers, vol. E3, no. 1, pp. 104-108, 2004.

[18] C. Perry, "The resistance strain gage revisited," Experimental Mechanics, vol. 24, no. 4, pp. 286-299, 1984.

[19] W. Thomson, Theory of Vibration with Applications 5th Edition, M. Dahleh, Ed. New Jersey: Prentice-Hall, Inc., 1998.

[20] Y. Jia and A. Seshia, "Five topologies of cantilever-based mems piezoelectric vibration energy harvesters: a numerical and experimental comparison," Microsyst. Technol., p. 12p, 2015.

[21] J. E.-Y. Lee, B. Bahreyni, and A. A. Seshia, "An axial strain modulated double-ended tuning fork electrometer," Sens. Actuators A, vol. 148, no. 2, pp. 395-400, 2008.

[22] M. Ferri, F. Mancarella, A. A. Seshia, J. H. T. Ransley, K. Soga, J. Zalesky, and A. Roncaglia, "Development of mems strain sensors for crack monitoring in ageing civil infrastructures," Smart Struct. Syst., vol. 6, no. 3, pp. 225-238, 2010. 
[23] M. Ferri, L. Belsito, F. Mancarella, L. Masini, A. Roncaglia, J. Yan, A. A. Seshia, J. Zalesky, and K. Soga, "Fabrication and testing of high resolution extensometer based on resonant mems strain sensors," in Transducers, Beijing, China, June 5-9 2011, p. 4.

[24] L. Belsito, M. Ferri, F. Mancarella, A. Roncaglia, J. Yan, A. A. Seshia, and K. Soga, "High resolution strain sensing on steel by silicon-on-insulator flexural resonators fabricated with chip-level vacuum packaging," in Transducers, Barcelona, Spain, 16-20 June 2013, p. 4.

[25] Y. Jia, J. Yan, K. Soga, and A. Seshia, "Parametrically excited mems vibration energy harvesters with design approaches to overcome initiation threshold amplitude," J. Micromech. Microeng, vol. 23, no. 11, p. 10pp., 2013.

[26] R. Zheng, K. Nakano, H. Hu, D. Su, and M. P. Cartmell, "An application of stochastic resonance for energy harvesting in a bistable vibrating system," J. Sound Vib, vol. 333, no. 12, pp. 2568-2587, 2014. 